\title{
BMJ Open Influence of hospital volume on nephrectomy mortality and complications: a systematic review and meta-analysis stratified by surgical type
}

\author{
Ray C J Hsu, ${ }^{1,2}$ Theodosia Salika, ${ }^{3}$ Jonathan Maw, ${ }^{2}$ Georgios Lyratzopoulos, ${ }^{3,4}$ \\ Vincent J Gnanapragasam, ${ }^{1,2}$ James N Armitage ${ }^{2}$
}

To cite: Hsu RCJ, Salika T, Maw J, et al. Influence of hospital volume on nephrectomy mortality and complications: a systematic review and meta-analysis stratified by surgical type. BMJ Open 2017;7:e016833. doi:10.1136/ bmjopen-2017-016833

- Prepublication history and additional material are available. To view these files please visit the journal online (http://dx.doi. org/10.1136/bmjopen-2017016833).

VJG and JNA contributed equally.

Received 14 March 2017 Revised 22 May 2017 Accepted 28 June 2017

\section{CrossMark}

${ }^{1}$ Academic Urology Group, Department of Surgery, University of Cambridge, Cambridge, UK

${ }^{2}$ Department of Urology, Addenbrooke's Hospital, Cambridge University Hospitals NHS Foundation Trust, Cambridge, UK

${ }^{3}$ Epidemiology of Cancer Healthcare and Outcomes(ECHO) Group, Department of Behavioural Science and Health, University College London, London, UK

${ }^{4}$ Cambridge Centre for Health Services Research, University of Cambridge, Cambridge, UK

Correspondence to

Dr Ray C J Hsu;

rch72@cam.ac.uk

\section{ABSTRACT}

Objectives The provision of complex surgery is increasingly centralised to high-volume (HV) specialist hospitals. Evidence to support nephrectomy centralisation however has been inconsistent. We conducted a systematic review and meta-analysis to determine the association between hospital case volumes and perioperative outcomes in radical nephrectomy, partial nephrectomy and nephrectomy with venous thrombectomy.

Methods Medline, Embase and the Cochrane Library were searched for relevant studies published between 1990 and 2016. Pooled effect estimates for nephrectomy mortality and complications were calculated for each nephrectomy type using the DerSimonian and Laird random-effects model. Sensitivity analyses were performed to examine the effects of heterogeneity on the pooled effect estimates by excluding studies with the heaviest weighting, lowest methodological score and most likely to introduce bias from misclassification of standardised hospital volume. Results Some 226372 patients from 16 publications were included in our review and meta-analysis. Considerable between-study heterogeneity was noted and only a few reported volume-outcome relationships specifically in partial nephrectomy or nephrectomy with venous thrombectomy. HV hospitals were correlated with a $26 \%$ and $52 \%$ reduction in mortality for radical nephrectomy ( $0 \mathrm{R} 0.74,95 \% \mathrm{Cl} 0.61$ to $0.90, \mathrm{p}<0.01)$ and nephrectomy with venous thrombectomy $(\mathrm{OR} 0.48,95 \% \mathrm{Cl}$ 0.29 to $0.81, p<0.01$ ), respectively. In addition, radical nephrectomy in HV hospitals was associated with an $18 \%$ reduction in complications $(\mathrm{OR} 0.82,95 \% \mathrm{Cl} 0.73$ to 0.92 , $\mathrm{p}<0.01)$. No significant volume-outcome relationship in mortality ( $0 \mathrm{R} 0.84,95 \% \mathrm{Cl} 0.31$ to $2.26, \mathrm{p}=0.73$ ) or complications ( $0 \mathrm{R} 0.85,95 \% \mathrm{Cl} 0.55$ to $1.30, \mathrm{p}=0.44$ ) was observed for partial nephrectomy.

Conclusions Our findings suggest that patients undergoing radical nephrectomy have improved outcomes when treated by HV hospitals. Evidence of this in partial nephrectomy and nephrectomy with venous thrombectomy is however not yet clear and could be secondary to the low number of studies included and the small patient number in our analyses. Further investigation is warranted to establish the full potential of nephrectomy centralisation particularly as existing evidence is of low quality with significant heterogeneity.
Strengths and limitations of this study

- This is a contemporary systematic review and metaanalysis of the associations between hospital case volumes and nephrectomy outcomes.

- Sixteen primary studies, which is fourfold greater in number than previous meta-analyses, were used to synthesise the pooled effect estimates for nephrectomy mortality and complications.

- To the best of our knowledge, this is the first study to date to stratify analyses based on nephrectomy type to account for differences in technical complexity and rates of adverse outcomes.

- Current evidence in nephrectomy outcome-volume relationship is of low quality and considerable heterogeneity exists between studies in design, type of data used, outcomes measured and statistical methodologies.

- Our study highlights the limitations in existing evidence and suggests questions that should be addressed in future research.

\section{INTRODUCTION}

In recent years, there has been an emerging trend for the centralisation of complex operations in healthcare systems around the world. ${ }^{1-3}$ This shift is supported by the growing research and evidence suggesting that hospitals and surgeons with high caseloads have better patient outcomes. ${ }^{4-8}$ Proponents argue that centralisation allows more effective use of clinical expertise and specialist equipment, and the increased exposure improves surgical skills and provides better training opportunities. Centralisation can also facilitate quicker adoption of care pathways, such as enhanced recovery, and may have more long-term financial sustainability for hospitals. However, surgical centralisation requires further travel distance and limits patient choice when many would prefer to undergo surgery locally even if greater mortality risks are taken into consideration. ${ }^{9}$ Differences in 
disease biology, surgical complexity and rate of adverse outcomes may also limit the perceived benefits of centralisation. Such a health service model may therefore not be appropriate for all conditions and operations.

Renal cancer accounts for over $2 \%$ of all new cancer diagnoses worldwide affecting more than 330000 individuals annually. ${ }^{10}$ Widespread use of cross-sectional imaging and increasing prevalence of obesity have contributed to a rising renal cancer incidence in many countries. ${ }^{11-13}$ Despite recent developments in systemic therapies, nephrectomy is often considered the only potentially curative treatment for renal cancer, and the number of nephrectomies being performed is likely to increase as a result. It is, therefore, critical that health service providers understand the effects that organisational changes may have on patient outcomes. While there has been an expansion of volume-outcome research, no consensus has so far been reached on the efficacy of centralising nephrectomy, and many uncertainties remain about its potential benefits particularly as radical and partial nephrectomy carry different surgical complexities and outcomes. ${ }^{14} 15$ We present a contemporary systematic review and meta-analysis of the published literature on the association between hospital case volumes and perioperative outcomes stratified by nephrectomy types. We hypothesise that outcomes significantly improve with higher nephrectomy case volumes.

\section{METHODS}

\section{Search criteria and data extraction}

The systematic review and meta-analysis was reported in accordance to the Preferred Reporting Items for Systematic Reviews and Meta-Analyses (PRISMA) guidelines (online supplementary appendix 1). ${ }^{16}$ Medical subject heading terms and key words for nephrectomy, case volume and outcomes were used in Medline, Embase and the Cochrane Library to search for relevant studies published between January 1990 and December 2016 (online supplementary appendix 2). Studies published prior to 1990 were not considered as recent medical and surgical advancements would have limited their applicability to the modern healthcare system. Only studies published in English were considered as the risk of potential language bias associated with this exclusion generally has little effect on summary effect estimates. ${ }^{17}$ References were searched manually for additional relevant studies.

We included studies that presented original data in fulltexts on adult nephrectomy outcomes across two or more hospital case volume categories. Abstracts, case reports and review articles were excluded(online supplementary appendix 3). No restriction was set on the study design and both prospective and retrospective studies were considered. Only those describing the volume-outcome relationships in radical nephrectomy with or without venous thrombectomy and/or partial nephrectomy were eligible. Paediatric cohorts were excluded as were articles comprised solely of nephroureterectomy or nephrectomy for non-oncological indications. Restriction on the reported outcomes was only applied at the end stage of the search to enable assessment of the current published evidence. Only studies reporting nephrectomy mortality and complications were included in the final analysis.

Two investigators (RCJH and JM) independently reviewed all studies for inclusion, data extraction and methodological quality. Any disagreement between the two reviewers was resolved by discussion and consultation with a third reviewer (JNA). Where only rates of outcomes were presented, these were applied to the case number to give the number of events, within the error of the published results. Study authors were contacted for further clarification if specific rates of outcomes and case numbers were not published. ${ }^{1819}$

As the cut-off values for hospital case volume categories differed among studies, we used the approach adopted by similar previous meta-analyses by dichotomising the volume groups presented by each study into low volume (LV) and high volume (HV) when the article presented an even number of volume groupings. ${ }^{20}{ }^{21}$ If a study presented an odd number of volume groups, the middle group was considered as LV.

Methodological quality and potential risk of bias were scored using a 10-domain system designed to measure the degree in which the study is likely to reveal generalisable conclusion about the magnitude and nature of the volume-outcome relationship. ${ }^{22}{ }^{23}$ Each domain provides a score between 0 and 3 with a total maximum of 18, suggesting a well-designed study. The parameters included the representativeness of the sample, the number of hospitals analysed, the samples size, the number of adverse events recorded, the appropriateness of patient selection, the number of volume categories examined, the number of outcomes measured, the degree of risk adjustment performed, whether hospital and surgeon case volumes were analysed in conjunction and whether clinical processes of care were measured.

If studies extracted data from the same source with overlaps in the study periods, we employed the following rules to avoid duplicating populations: (1) studies with identical patient cohort but examining different outcomes were considered and analysed separately, (2) studies that derived data from older datasets were excluded in favour of the more contemporary cohort, (3) if the above rules were not applicable, studies with the lower methodological quality scores were excluded and (4) where quality scores were equal, the study covering the longest period was included.

\section{Quantitative data synthesis}

All statistical analyses were performed using Stata $14 .{ }^{24}$ Nephrectomy types were categorised into radical nephrectomy, partial nephrectomy and nephrectomy with venous thrombectomy and analysed separately. Studies involving multiple types of nephrectomies were analysed based on the aforementioned groups, but if this was not feasible, they were categorised as radical nephrectomy. With the 
assumption that a distribution of effects exists among studies, all pooled effect size were calculated using the DerSimonian and Laird random-effects model, which provided more conservative estimates compared with fixed-effect model. OR and 95\% CI were calculated and presented for each outcome measure using LV groups as the reference.

When the meta-analysis demonstrated significantly better outcomes in HV hospitals, we quantified the clinical effectiveness of centralisation by calculating the numbers needed to treat, or in our case numbers needed to centralise (NNC). NNC represents the number of cases that will need to be centralised from LV hospitals and treated by HV hospitals in order to prevent one adverse event.

\section{Heterogeneity}

As the DerSimonian and Laird model would have only accounted for some between-study heterogeneity, we further quantified heterogeneity by calculating $\mathrm{I}^{2}$ statistic. $\mathrm{I}^{2}$ provides an easily understood number, which describes the proportion of total variation in estimates that is due to heterogeneity rather than chance..$^{25}$ Values of $25 \%$ or lower denote low heterogeneity and values of $75 \%$ or greater denote considerable heterogeneity. ${ }^{26}$ Meta-regression was performed to explore the influence of potential explanatory variables on heterogeneity including each study's publication year, country, data source, number of patients and their demographics, number of hospitals and threshold for HV hospitals.

\section{Publication bias}

Funnel plots were generated to investigate potential publication bias, and were enhanced to include contours that divide the funnel into statistically significant and non-significant areas. Funnel plot symmetry suggests low probability of publication bias and Harbord's modified test was used to test for asymmetry. ${ }^{27}$ Harbord's test reduces false positive rates when applied to binary outcome data, especially when there is low between-study heterogeneity.

Trim and fill method was also performed to account for publication bias by adjusting the meta-analysis to incorporate the theoretically missing studies. ${ }^{28}$

\section{Sensitivity analysis}

To examine specific studies' effects on pooled effect size, sensitivity analyses were performed by excluding individual studies and repeating the meta-analyses. We examined the effects of studies with the heaviest weighting and studies with the lowest methodological quality score. As there is currently no consensus on what nephrectomy case volume is necessary to be considered as $\mathrm{HV}$, we repeated our analyses by excluding studies whose standardised HV categories overlapped most significantly with the standardised LV categories in other studies to account for potential bias of misclassifying volume categories in our dichotomy.
As secondary analyses, we additionally repeated the meta-analysis three further times with different methods of dichotomising the volume groups to examine whether our initial estimates would remain consistent. The methods of dichotomising were (1) lowest volume categories and all others, (2) even dichotomy and when studies present an odd number of volume categories, the middle group was considered as HV and (3) highest volume categories and all others.

\section{RESULTS}

\section{Study selection and characteristics}

From the 5680 articles initially identified, 16 were included in the systematic review containing 226372 patients from six countries (figure 1). For the meta-analysis, 11 studies with 201506 patients examining radical nephrectomy were included while 4 studies of 23617 patients and 2 studies of 1249 patients examining partial nephrectomy and nephrectomy with venous thrombectomy were included, respectively. Publication year ranged from 2002 to 2016, while cohort periods covered from 1993 to 2013.

Table 1 summarises the characteristics of the included studies. Variations were observed in study designs including source of data and outcomes measured. Out of a maximum possible score of 18 , the median quality score from the included studies was nine (IQR 8-9) with the majority of the studies failing to adequately address potential confounders including measuring the appropriateness of patient selection, adjusting for case-mix variations and accounting for differences in clinical risks and processes of care (online supplementary appendix 4). Variable thresholds for HV hospitals were noted across the included studies.

\section{Mortality and hospital volumes}

Postoperative mortality, defined as inpatient or 30 day, was the most frequently examined outcome reported in 14 studies. Ten studies reported mortality in radical nephrectomy, ${ }^{18-37}$ three in partial nephrectomy ${ }^{36} 3839$ and two in nephrectomy with venous thrombectomy. ${ }^{19} 40$ The overall mortality was $1.59 \%$ (range $0.20 \%-7.2 \%$ ) with mortality rates in HV and LV hospitals being $1.47 \%$ and $1.68 \%$, respectively.

\section{Radical nephrectomy}

Meta-analysis demonstrated that patients who underwent radical nephrectomy in HV hospitals had a $26 \%$ reduction (OR $0.74,95 \%$ CI 0.61 to $0.90, \mathrm{p}<0.01$ ) in postoperative mortality, corresponding to an NNC of 234 (figure 2A).

Significant heterogeneity was observed $\left(\mathrm{I}^{2}=75.0 \%\right.$, $\mathrm{p}<0.01)$. Meta-regression was performed to investigate the potential explanatory variables for heterogeneity, and only differences in the threshold values for HV hospitals were shown to be a significant contributor (online supplementary appendix 5A). Subgroup analysis of the three studies examining exclusively radical nephrectomies 


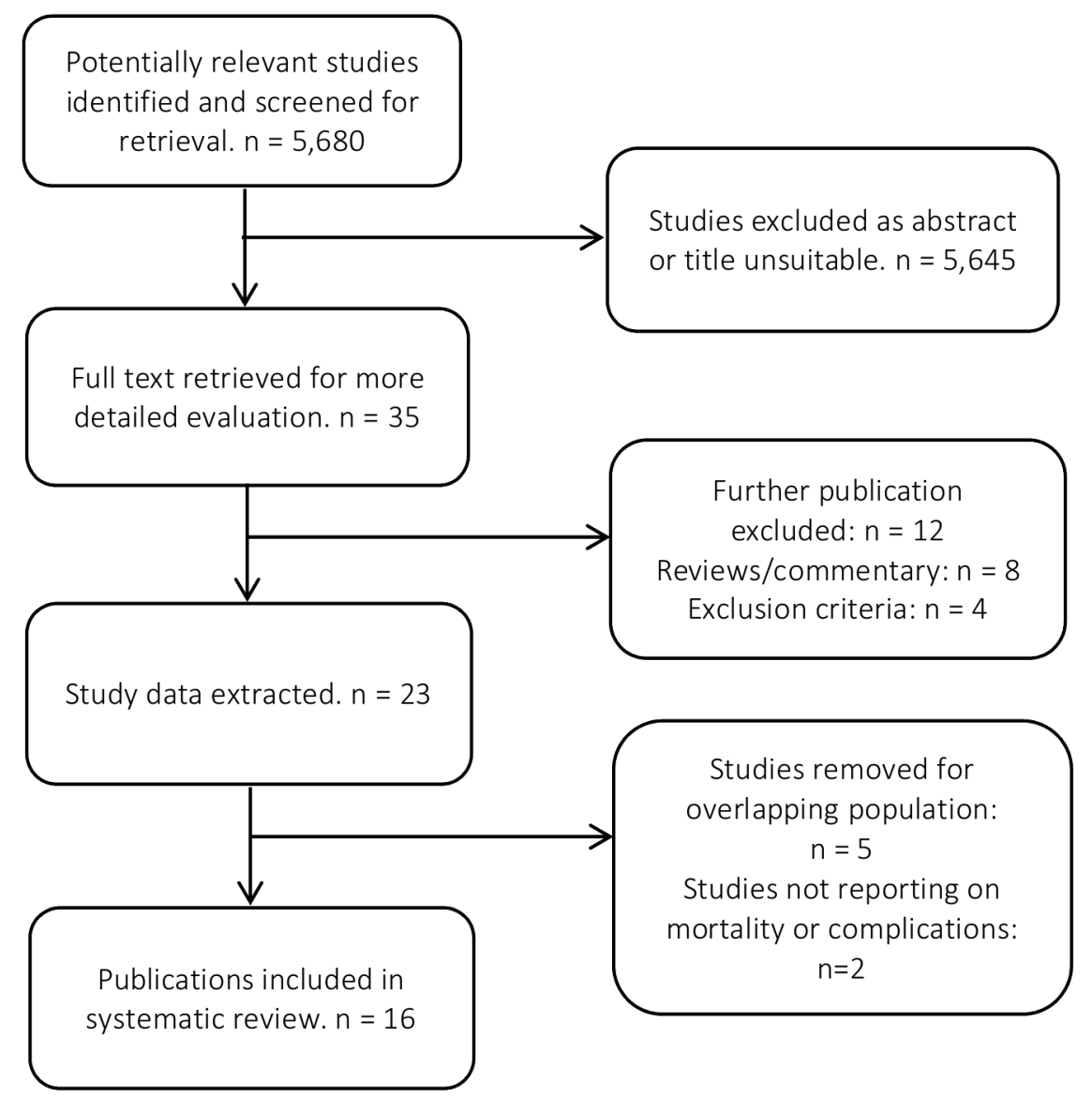

Figure 1 Flow chart of the article selection process.

demonstrated a more pronounced reduction in postoperative mortality favouring $\mathrm{HV}$ hospitals (OR $0.62,95 \% \mathrm{CI}$ 0.53 to $0.71, \mathrm{p}<0.01) .{ }^{18}{ }^{34} 36$ This corresponded to a lower NNC of 166 with little residual heterogeneity $\left(I^{2}=0.0 \%\right.$, $\mathrm{p}=0.40$ ). The overall funnel plot was visually asymmetrical particularly missing studies with effect estimates favouring LV hospitals (figure 3). However, Harbord's modified test did not show significant asymmetry $(\mathrm{p}=0.40)$ and 'trim and fill' method did not change the initial estimate, indicating no clear evidence of publication bias.

In sensitivity analyses, exclusion of the most heavily weighted study led to a similar pooled-effect estimate (OR $0.70,95 \%$ CI 0.55 to $0.88, \mathrm{p}<0.01) .{ }^{37}$ Exclusion of the study with the lowest quality score also did not significantly alter our result (OR 0.74, 95\% CI 0.61 to $0.91, \mathrm{p}<0.01) .{ }^{35}$ To examine the potential bias introduced by misclassification of hospital volume, two cohorts were excluded..$^{303135}$ This did not substantially change our pooled-effect estimate either (OR $0.73,95 \%$ CI 0.58 to $0.93, \mathrm{p}=0.01$ ). Overall, radical nephrectomies in HV hospitals appeared to have significantly lower mortality.

\section{Partial nephrectomy}

Meta-analysis showed that partial nephrectomy patients operated in $\mathrm{HV}$ hospitals had a $16 \%$ reduction in postoperative mortality, but this was not statistically significant (OR 0.84, 95\% CI 0.31 to 2.26, $\mathrm{p}=0.73$ ) (figure 2B). Moderate but non-significant heterogeneity was noted $\left(\mathrm{I}^{2}=36.84 \%, \mathrm{p}=0.21\right)$. Sensitivity analyses removing studies with the heaviest weighting ${ }^{39}$ or most likely to introduce misclassification bias of exposure ${ }^{38}$ demonstrated reduced mortality favouring HV hospitals, but these remained non-significant.

Nephrectomy with venous thrombectomy

Patients who underwent nephrectomy with venous thrombectomy in $\mathrm{HV}$ hospitals had a $52 \%$ reduction (OR $0.48,95 \%$ CI 0.29 to $0.81, \mathrm{p}<0.01$ ) in short-term mortality compared with $\mathrm{LV}$ hospitals (figure $2 \mathrm{C}$ ). This corresponded to an NNC of 25 with low heterogeneity $\left(\mathrm{I}^{2}=0.0 \%, \mathrm{p}=0.50\right)$. Due to the small number of studies reporting this outcome, further testing of heterogeneity and publication bias was not expected to generate meaningful results and this was not attempted.

\section{Complications and hospital volumes}

Complication was the second most frequently investigated outcome, reported in 11 studies. Events considered as a complication differed among studies (table 1). Seven studies reported complications in radical 


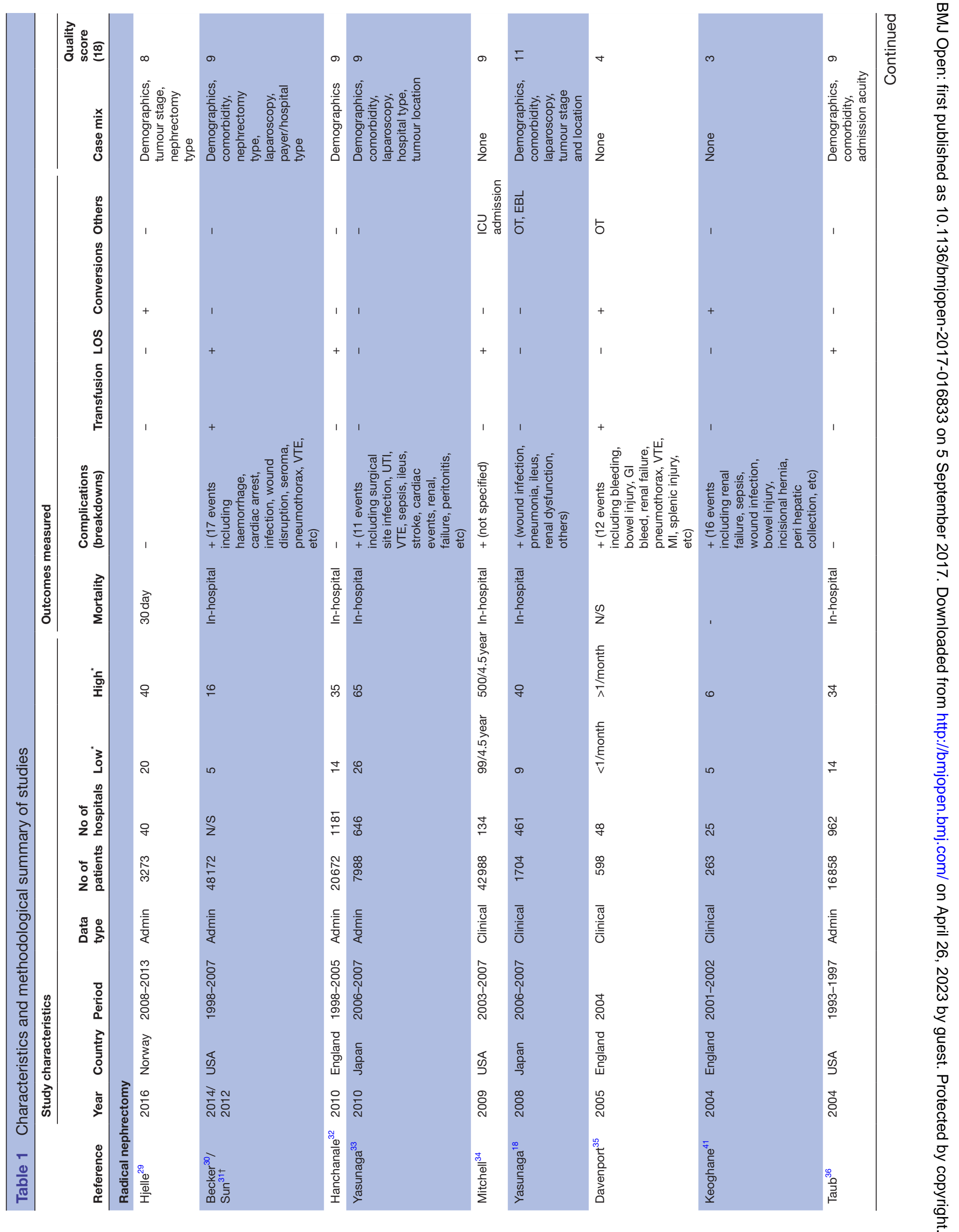




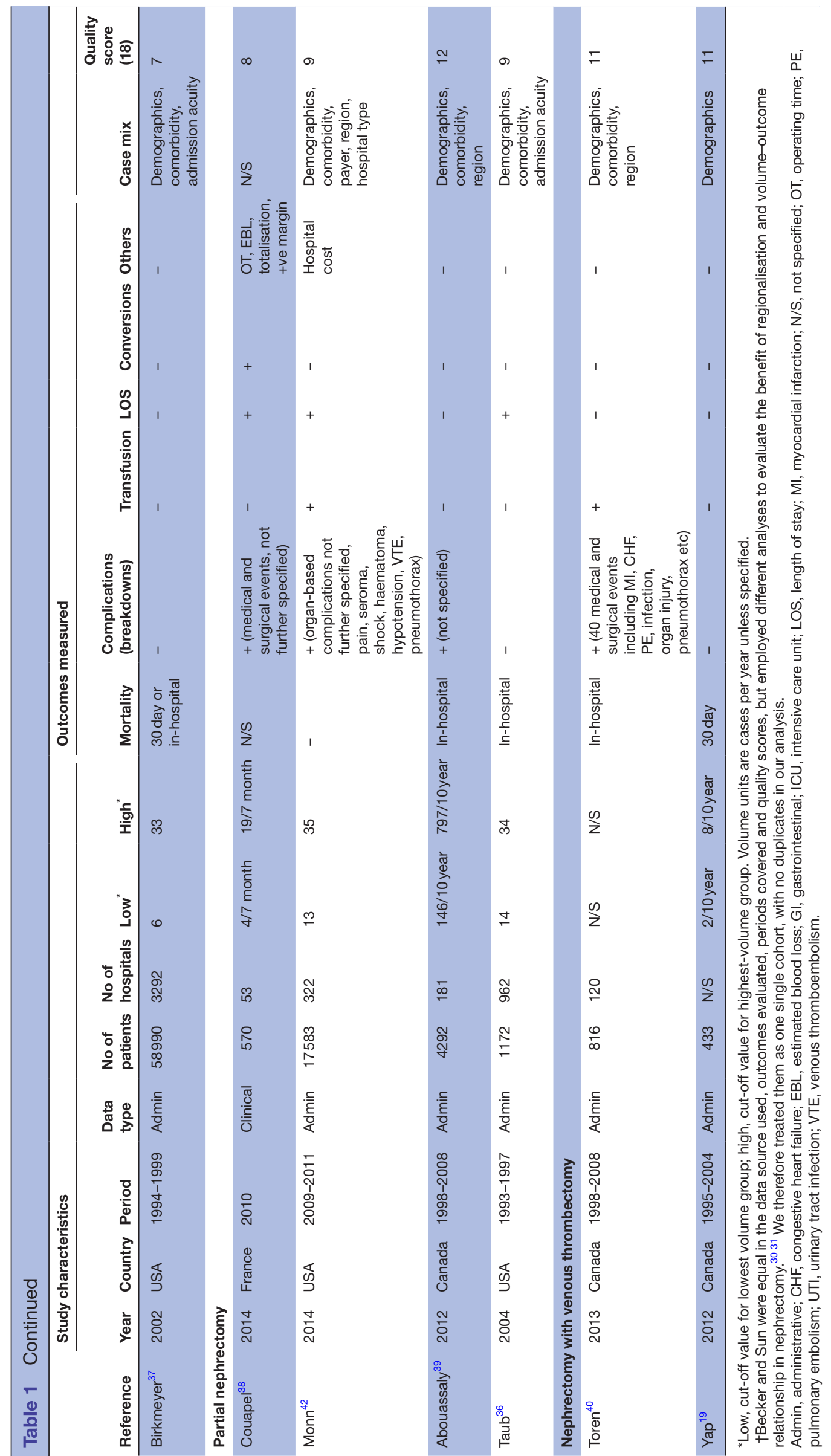



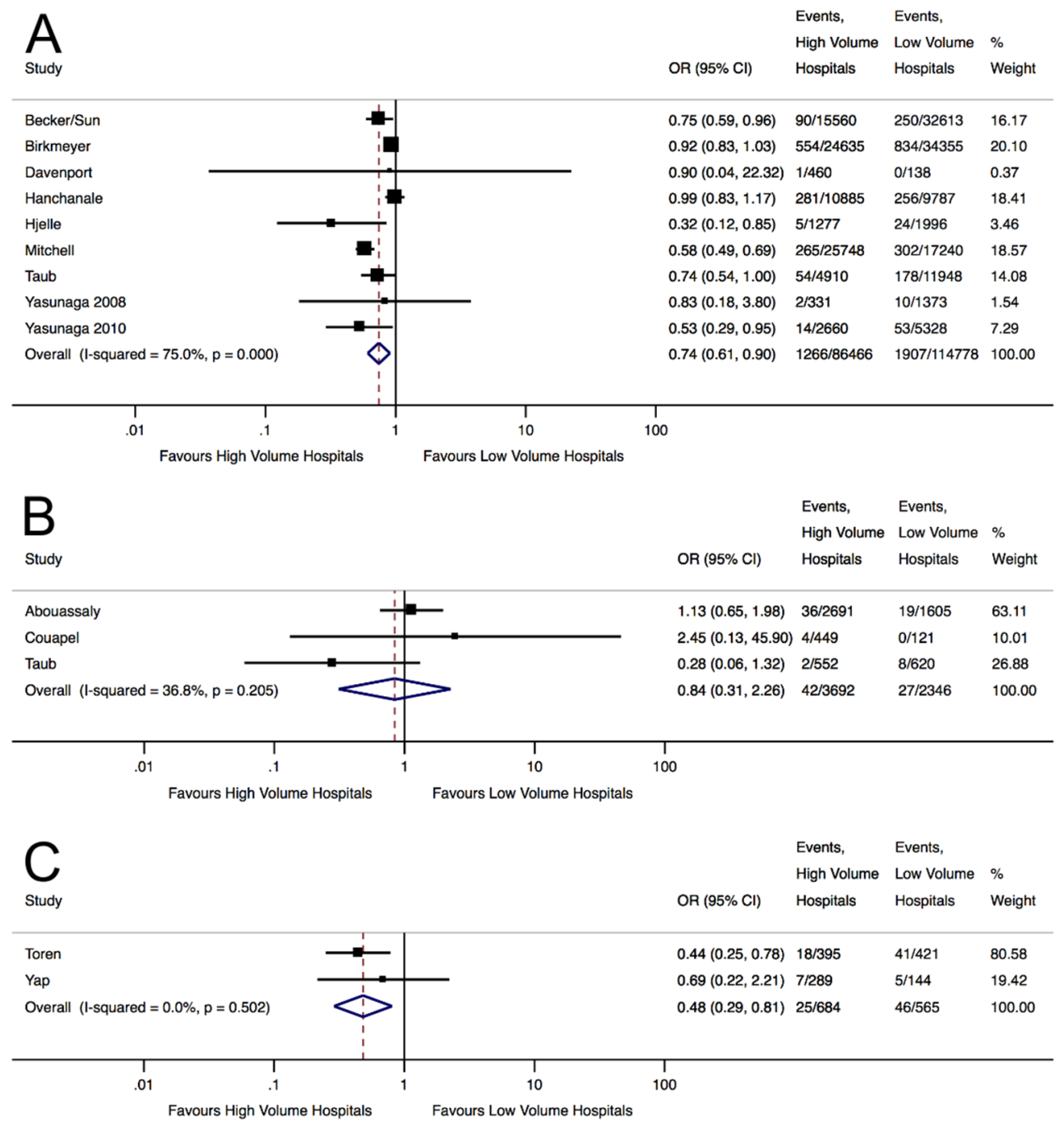

Figure 2 Forest plots displaying the pooled effect estimates of nephrectomy mortality in high-volume and low-volume hospitals for $(A)$ radical nephrectomy, (B) partial nephrectomy and (C) nephrectomy with venous thrombectomy.

nephrectomy ${ }^{18} 3031$ 33-35 41 and three in partial nephrectomy. ${ }^{39} 32$ Only one study examined volume-outcome relationship in nephrectomy with venous thrombectomy and meta-analysis was, therefore, not appropriate. ${ }^{40}$ The overall complication rate was $16.26 \%$ (range $7.4 \%-78 \%$ ). HV hospitals had complication rates of $15 \%$ compared with $17.51 \%$ in LV hospitals.

\section{Radical nephrectomy}

Meta-analysis showed an $18 \%$ reduction (OR $0.82,95 \%$ CI 0.73 to $0.92, \mathrm{p}<0.01)$ in nephrectomy complications in $\mathrm{HV}$ centres, corresponding to an NNC of 38 (figure 4A). Significant heterogeneity was noted $\left(\mathrm{I}^{2}=76.25 \%, \mathrm{p}<0.01\right)$, but none of the factors examined in meta-regression significantly contributed to this (online supplementary appendix 5B). Sensitivity analyses by removing studies with the lowest quality ${ }^{41}$ or most likely to introduce misclassification bias of exposure ${ }^{35} 41$ did not significantly alter our initial result. Excluding study with the heaviest weighting however led to a loss of significance in the pooled-effect estimate, which however still demonstrated a $11 \%$ reduction in complications in HV hospitals
(OR $0.89,95 \%$ CI 0.74 to $1.08, \mathrm{p}=0.24$ ). Overall, radical nephrectomies performed in HV hospitals appeared to have significantly lower complications compared with LV hospitals.

\section{Partial nephrectomy}

Partial nephrectomy patients operated in HV hospitals had a $15 \%$ reduction in complications, but this was not statistically significant (OR 0.85 , 95\% CI 0.55 to 1.30 , $\mathrm{p}=0.44$ ) (figure 4B). Significant heterogeneity was noted $\left(\mathrm{I}^{2}=94.80 \%, \mathrm{p}<0.01\right)$. Sensitivity analysis by removing studies with the heaviest weighting ${ }^{42}$ or most likely to introduce misclassification bias of hospital volume ${ }^{38}$ did not result in significance.

\section{Secondary analyses using different methods for dichotomising HV and LV}

As there is no consensus on what constituted HV hospitals in current evidence, simple dichotomy of volume groups may introduce inherent bias to the estimates. Yet no recommendation on how best to proceed in volumeoutcome analysis presently exists. 


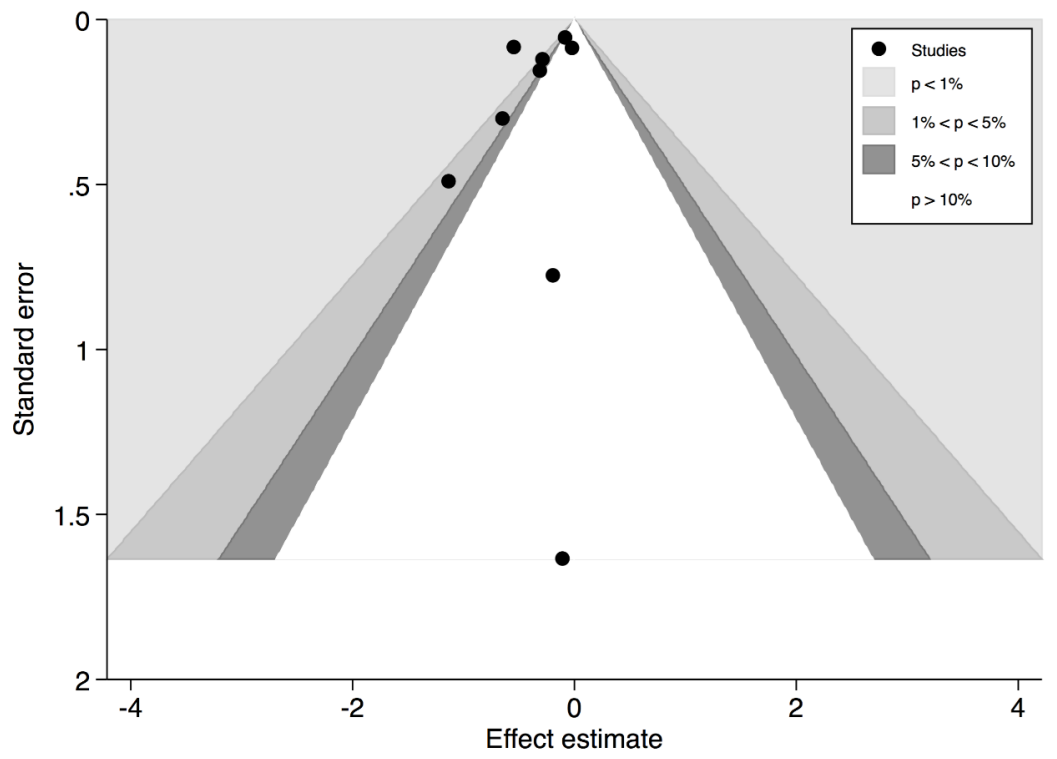

Figure 3 Contour-enhanced funnel plot of studies analysing hospital volume-outcome relationship in radical nephrectomy mortality. Harbord's modified test for funnel plot asymmetry was not statistically significant.

In our secondary analyses, we consistently observed significantly lower risks of mortality for both radical nephrectomy and nephrectomy with venous thrombecotmy in HV hospitals regardless how volumes were dichotomised (table 2). The magnitudes of risk reductions were more pronounced when higher thresholds for HV hospitals were considered particularly for radical nephrectomy mortality. Partial nephrectomy mortality however continued to demonstrate no significant association to volume even when dichotomies were comparing the highest volume groups to all others.

Risks of radical nephrectomy complications remained significantly reduced in HV hospitals when the dichotomy threshold for HV hospitals was increased, but the significance was lost when the thresholds were lowered. Association between hospital volumes and partial nephrectomy complications remained insignificant regardless of how HV was defined in our dichotomy.

\section{DISCUSSION}

Evidence on volume-outcome relationships in complex diseases and procedures has increased substantially in recent years. Many operations have been shown to have improved outcomes in HV centres, but this may not be uniform across all surgeries and benefits have not been
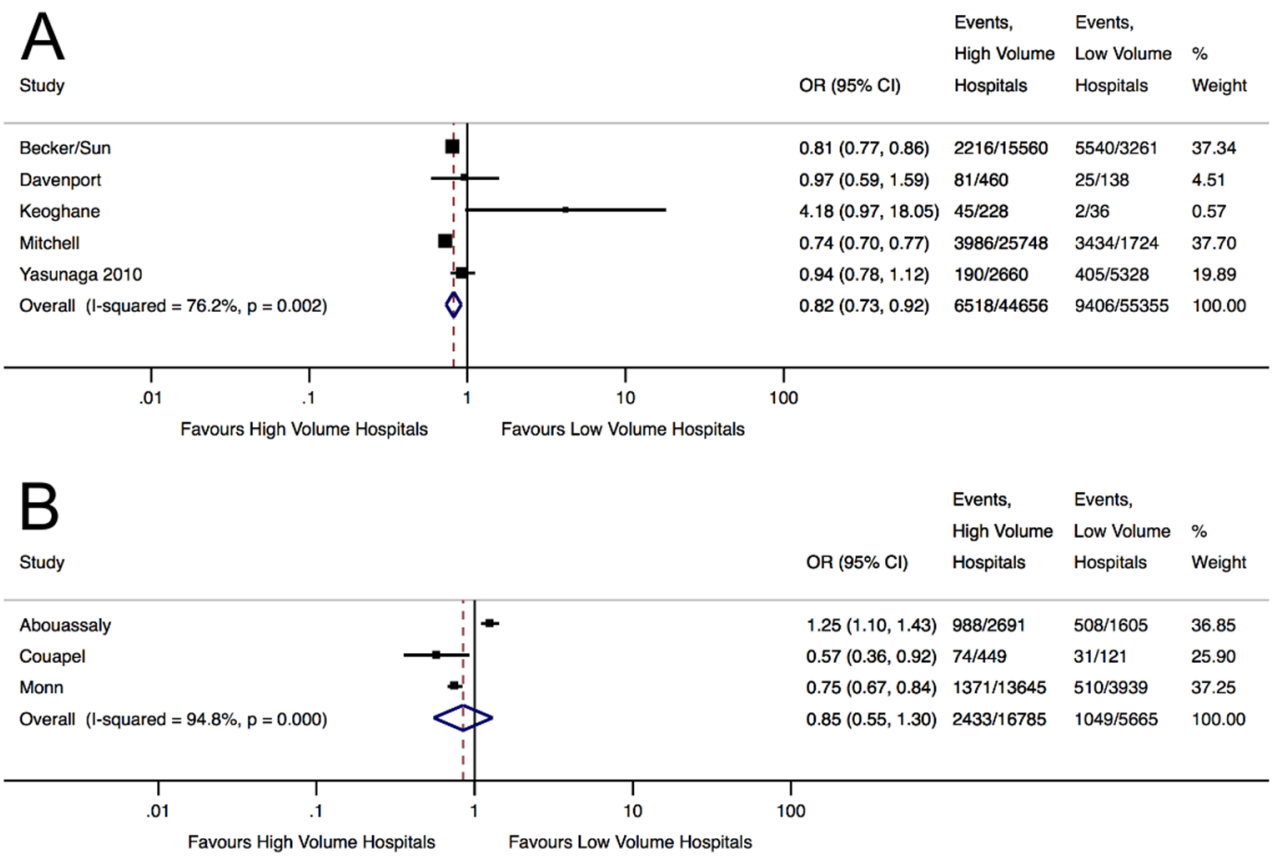

Figure 4 Forest plots displaying the pooled effect estimates of nephrectomy complications in HV and LV hospitals for (A) radical nephrectomy and (B) partial nephrectomy. 
Table 2 Results of meta-analysis by using different methods of dichotomising volume groups

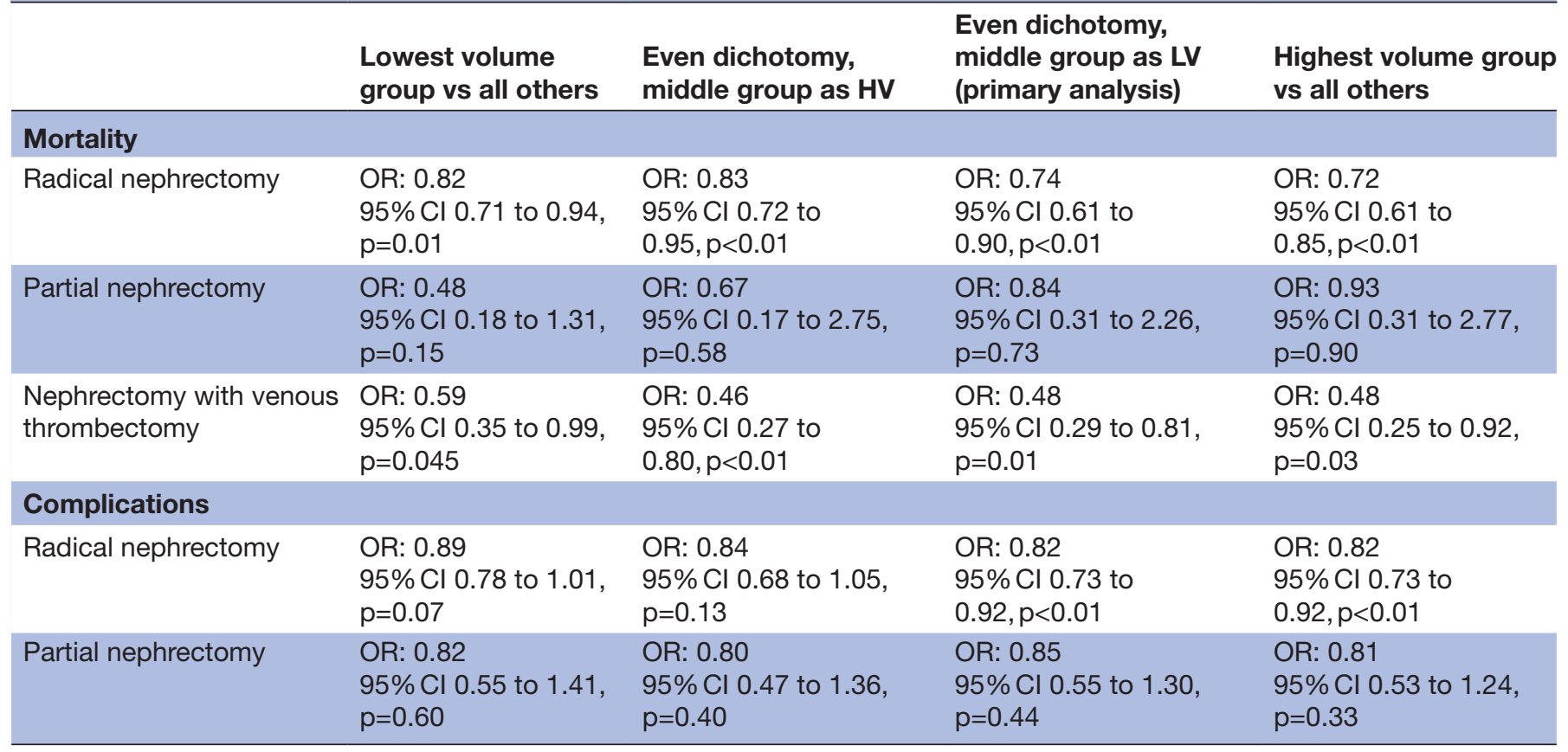

$\mathrm{HV}$, high volume; LV, low volume.

associated with volume in percutaneous nephrolithotomy or appendicectomy. ${ }^{43-48}$ This meta-analysis provides a contemporary review of the effects of centralisation in nephrectomy outcomes. It reveals significant inverse associations between hospital case volumes for short-term mortality and complications for radical nephrectomy, but evidence of these for partial nephrectomy and nephrectomy with venous thrombectomy remains less compelling.

Considered individually, all but three studies in our review reported lack of associations between hospital volume and nephrectomy mortality. ${ }^{29} 3637$ However, such associations in favour of $\mathrm{HV}$ hospitals were apparent when considering the totality of the evidence particularly in radical nephrectomy and venous thrombectomy. This finding is consistent with the only other meta-analysis on nephrectomy volume-outcome relationship published in 2009 but includes fourfold greater number of studies. ${ }^{23}$ Our meta-analysis demonstrates that the mortality benefit seen in radical nephrectomy may be relatively small requiring centralisation of 234 patients in order to avoid one death. However, the NNC decreased considerably to 166 in our sensitivity analyses. Coupled with the much lower NNC of 38 for radical nephrectomy complications, there is moderate evidence to support its centralisation.

In our analyses, the mortality reduction for venous thrombectomy was observed to be more pronounced than that in radical nephrectomy. This is consistent with the 'practice-makes-perfect' hypothesis particularly as venous thrombectomy is a technically more challenging procedure compared with radical nephrectomy, though interestingly a similar trend was not observed for partial nephrectomy. ${ }^{49}$ These results should however be interpreted taking into consideration that only a few studies have so far reported on the volume-outcome relationships for partial nephrectomy and venous thrombectomy and the pooled effect estimates were synthesised from just two to three publications, thus the overall evidence is weak. As partial nephrectomy has only been widely adopted in the last two decades and nephrectomy centralisation also a relatively recent phenomenon, it is likely that more evidence will emerge in the coming years and repeating the meta-analysis at such point is warranted. ${ }^{150}$ This will be of particular importance as partial nephrectomy has been demonstrated to be a safe procedure and the relatively small number of partial nephrectomy patients in our meta-analysis might not have been sufficiently powered to reveal the true presence of a volumeoutcome relationship, as evident in our wide CI. ${ }^{51}$ This may also explain the lack of significant association between partial nephrectomy complications and hospital volumes. With its low mortality and morbidity rates, other outcome measures such as ischaemic time and negative surgical margins, are likely to be more appropriate quality markers in volume-outcome analysis, but these have so far been poorly evaluated.

Despite the strict inclusion criteria in our studies, we observed considerable heterogeneity, especially in the meta-analyses of nephrectomy complications. One explanation for this is the lack of standardised reporting of complications by individual studies. Harder endpoints as previously discussed could have overcome this. Other more objective outcomes including transfusion rate and length of stay were reported by four ${ }^{30} 313542$ and seven studies $^{30-3234384252}$ in our systematic review, respectively, but they were not in adequate numbers to be stratified by nephrectomy types or in sufficiently detailed data to 
perform meta-analyses. In addition, variations in the threshold values for HV hospitals likely contributed to the heterogeneity, although this was not evident in the meta-regression. Volume was also used as a proxy marker for surgical and care quality, but the precise clinical processes that may improve patient outcomes were not directly measured or identified. It is therefore conceivable for some heterogeneity to arise from these unmeasured practices. Results from the multiple sensitivity analyses to adjust for study differences have however remained robust and our study would still appear to be informative and relevant. There are other research designs that may be more appropriate in testing our hypothesis such as analysis of primary data amalgamated from multiple population cohorts. The considerable ethical concerns and logistical constraints of this may however be challenging to overcome and not practically feasible.

While there has been an expansion in the studies on nephrectomy volume-outcome relationship, many questions continue to be unanswered. The proportion of nephrectomy performed under laparoscopy or robotic assistance is growing. ${ }^{53} 54$ There is however a paucity of evidence specifically investigating this in the volume-outcome context with only one study examining the differences in perioperative measures in robotic partial nephrectomy. ${ }^{42}$ Three other studies have adjusted surgical techniques in multivariable regressions, but these did not directly demonstrate the effect of laparoscopic volumes on surgical outcomes. ${ }^{18} 3033$ Due to the small study number and data quality, it was not possible in this meta-analysis to further substratify each nephrectomy type into open and minimally invasive and our results should be interpreted taking this limitation into account.

Tumour characteristics including TNM stage and grades are well established to significantly affect and predict nephrectomy mortality, but only two studies have so far adjusted for this in their analyses. ${ }^{18} 29$ Surgeon case volume and degree of specialisation also play significant roles in determining operative outcome, and can be more important than hospital case volume alone ${ }^{45}{ }^{55-57}$ While not the focus of this study, no significant association was found between surgeon volume and complications in radical nephrectomy, ${ }^{18}$ but $31 \%$ and $16 \%$ reduction in mortality and complications respectively was observed in partial nephrectomy in HV surgeons. ${ }^{39} \mathrm{HV}$ surgeons performing nephrectomy with venous thrombectomy were also reported to have reduced risk of mortality, ${ }^{19}$ but this was not observed in a subsequent study. ${ }^{40}$ As our analyses were based on crude pooled effect estimates, future meta-analysis should ideally attempt to adjust for other possible confounders including patient demographics, socioeconomic status and comorbidities, although this may be methodologically challenging. It would be of high interest to understand the interactions among patient characteristics, surgeon volume, surgical approach and oncological factors in the volume-outcome relationship and may provide additional insights to selecting patients that will benefit the most from nephrectomy centralisation, such as those with multiple comorbidities or advanced disease. Similarly, no study has examined the long-term benefits of centralising nephrectomy when HV centres have been demonstrated to increase oncological survival in other cancer surgeries. ${ }^{58}{ }^{59}$ Results of this may further influence the recommendations for nephrectomy centralisation and this is currently being explored in our ongoing work. Other outcomes including long-term risks of chronic kidney disease and cardiovascular morbidities and patient reported outcome measures may also provide more relevant and holistic measurements of the potential efficacy of nephrectomy centralisation.

Our secondary analyses would suggest that a minimum volume threshold for nephrectomy likely exist, and beyond that, risks of adverse outcomes may continue to decrease with further increase in volume. An important limitation of this however is that this minimum threshold cannot be objectively determined from the current evidence. The specific care processes that may produce good outcomes, such as access to nurse specialists and clinical trials, could not be determined from our study. As volume is likely to be a proxy marker for quality, increasing volume alone in itself is unlikely to reduce adverse results. ${ }^{60}$ Future research should concentrate on identifying the qualitative differences between providers in order for the contributing good practices to be adopted by lesser performing centres.

\section{CONCLUSIONS}

Current evidence of the association between hospital volumes and nephrectomy outcomes is of low quality with considerable between-study heterogeneity. Our meta-analyses demonstrated significant reductions in mortality and complications for patients undergoing radical nephrectomy in HV hospitals. Evidence of this in partial nephrectomy and nephrectomy with venous thrombectomy is not yet clear but warrants further investigations.

Acknowledgements We acknowledge Annie Herbert for providing valuable critiques of the methodologies.

Contributors We confirm that each author has participated sufficiently in the submission and the submission has been approved by all authors. RCJH, VJG and JNA contributed to the study conception and design. RCJH, JM and JNA contributed to data acquisition and risk of bias assessment. RCJH, TS, GL, VJG, JNA contributed to statistical analysis, data interpretation and critical manuscript revision. RCJH contributed to drafting the manuscript. RCJH contributed to obtain funding. GL, VJG and JNA contributed to supervision of the study.

Funding This work was supported by Addenbrooke's Charitable Trust, the Royal College of Surgeons of England and the Urology Foundation. Cancer Research UK Advanced Clinician Scientist Fellowship award (No. C18081/A18180) has supported GL and TS.

Competing interests None declared. 
Provenance and peer review Not commissioned; externally peer reviewed.

Data sharing statement № additional data are available.

Open Access This is an Open Access article distributed in accordance with the Creative Commons Attribution Non Commercial (CC BY-NC 4.0) license, which permits others to distribute, remix, adapt, build upon this work non-commercially, and license their derivative works on different terms, provided the original work is properly cited and the use is non-commercial. See: http://creativecommons.org/ licenses/by-nc/4.0/

(C) Article author(s) (or their employer(s) unless otherwise stated in the text of the article) 2017. All rights reserved. No commercial use is permitted unless otherwise expressly granted.

\section{REFERENCES}

1. National Institute for Clinical Excellence. Guidance on cancer services: improving outcomes in urological cancers. London, 2002

2. Cooperberg MR, Modak S, Konety BR. Trends in regionalization of inpatient care for urological malignancies, 1988 to 2002. J Urol 2007;178:2103-8.

3. Boudourakis LD, Wang TS, Roman SA, et al. Evolution of the surgeon-volume, patient-outcome relationship. Ann Surg 2009;250:159-65.

4. Birkmeyer JD, Lucas FL, Wennberg DE. Potential benefits of regionalizing Major surgery in Medicare patients. Eff Clin Pract 1999;2:277-83.

5. Nguyen NT, Paya M, Stevens CM, et al. The relationship between hospital volume and outcome in bariatric surgery at academic medical centers. Ann Surg 2004;240:184-92.

6. Pieper D, Mathes T, Neugebauer E, et al. State of evidence on the relationship between high-volume hospitals and outcomes in surgery: a systematic review of systematic reviews. J Am Coll Surg 2013;216:1015-25

7. Trinh QD, Bjartell A, Freedland SJ, et al. A systematic review of the volume-outcome relationship for radical prostatectomy. Eur Urol 2013;64:786-98.

8. Hounsome LS, Verne J, McGrath JS, et al. Trends in operative caseload and mortality rates after radical cystectomy for bladder cancer in England for 1998-2010. Eur Urol 2015;67:1056-62.

9. Finlayson SR, Birkmeyer JD, Tosteson AN, et al. Patient preferences for location of care: implications for regionalization. Med Care 1999;37:204-9.

10. Ferlay J, Soerjomataram I, Ervik M, et al. GLOBOCAN 2012 v1.0, Cancer Incidence and Mortality Worldwide: IARC CancerBase No. 11. 2013.. http://globocan.iarc.fr (accessed 13 Feb 2017)

11. Hollingsworth JM, Miller DC, Daignault S, et al. Rising incidence of small renal masses: a need to reassess treatment effect. J Nat Cancer Inst 2006;98:1331-4.

12. Chow WH, Dong LM, Devesa SS. Epidemiology and risk factors for kidney cancer. Nat Rev Urol 2010;7:245-57.

13. Znaor A, Lortet-Tieulent J, Laversanne M, et al. International variations and trends in renal cell carcinoma incidence and mortality. Eur Urol 2015;67:519-30.

14. Tan HJ, Norton EC, Ye Z, et al. Long-term survival following partial vs radical nephrectomy among older patients with early-stage kidney cancer. JAMA 2012;307:1629-35.

15. Kim SP, Thompson RH, Boorjian SA, et al. Comparative effectiveness for survival and renal function of partial and radical nephrectomy for localized renal tumors: a systematic review and meta-analysis. J Urol 2012;188:51-7.

16. Moher D, Liberati A, Tetzlaff J, et al. Preferred reporting items for systematic reviews and meta-analyses: the PRISMA statement. PLoS Med 2009;6:e1000097.

17. Jüni $P$, Holenstein $F$, Sterne J, et al. Direction and impact of language Bias in meta-analyses of controlled trials: empirical study. Int $J$ Epidemiol 2002;31:115-23.

18. Yasunaga $\mathrm{H}$, Yanaihara $\mathrm{H}$, Fuji $\mathrm{K}$, et al. Influence of hospital and surgeon volumes on operative time, blood loss and perioperative complications in radical nephrectomy. Int J Urol 2008;15:688-93.

19. Yap SA, Horovitz D, Alibhai SM, et al. Predictors of early mortality after radical nephrectomy with renal vein or inferior vena cava thrombectomy - a population-based study. BJU Int 2012;110:1283-8.

20. Awopetu Al, Moxey P, Hinchliffe RJ, et al. Systematic review and meta-analysis of the relationship between hospital volume and outcome for lower limb arterial surgery. Br J Surg 2010;97:797-803.

21. Holt PJ, Poloniecki JD, Loftus IM, et al. Meta-analysis and systematic review of the relationship between hospital volume and outcome following carotid endarterectomy. Eur J Vasc Endovasc Surg 2007;33:645-51.

22. Halm EA, Lee $C$, Chassin MR. Is volume related to outcome in health care? A systematic review and methodologic critique of the literature. Ann Intern Med 2002;137:511-20.

23. Mayer EK, Purkayastha S, Athanasiou T, et al. Assessing the quality of the volume-outcome relationship in uro-oncology. BJU Int 2009;103:341-9.

24. StataCorp. Stata Statistical Software: release 14. 2015.

25. Higgins JP, Thompson SG. Quantifying heterogeneity in a metaanalysis. Stat Med 2002;21:1539-58.

26. Higgins JP, Thompson SG, Deeks JJ, et al. Measuring inconsistency in meta-analyses. BMJ 2003;327:557-60.

27. Harbord RM, Egger M, Sterne JA. A modified test for small-study effects in meta-analyses of controlled trials with binary endpoints. Stat Med 2006;25:3443-57.

28. Duval S, Tweedie R. A Nonparametric "Trim and Fill" Method of Accounting for Publication Bias in Meta-Analysis. J Am Stat Assoc 2000;95:89-98.

29. Hjelle KM, Johannesen TB, Beisland C. Postoperative 30-day Mortality Rates for Kidney Cancer are dependent on Hospital Surgical volume: results from a norwegian Population-based study. Eur Urol Focus 2016:1-8.

30. Becker A, Bianchi M, Hansen J, et al. Benefit in regionalization of care for patients treated with nephrectomy: a nationwide inpatient sample. World J Urol 2014;32:1511-21.

31. Sun M, Bianchi M, Trinh QD, et al. Hospital volume is a determinant of postoperative complications, blood transfusion and length of stay after radical or partial nephrectomy. J Urol 2012;187:405-10.

32. Hanchanale VS, Javlé P. Impact of hospital provider volume on outcome for radical urological Cancer surgery in England. Urol Int 2010;85:11-15.

33. Yasunaga $\mathrm{H}$, Yanaihara $\mathrm{H}$, Fuji $\mathrm{K}$, et al. Impact of hospital volume on postoperative complications and in-hospital mortality after renal surgery: data from the Japanese Diagnosis Procedure Combination database. Urology 2010;76:548-52.

34. Mitchell RE, Lee BT, Cookson MS, et al. Radical nephrectomy surgical outcomes in the University HealthSystem Consortium Data Base: impact of hospital case volume, hospital size, and geographic location on 40,000 patients. Cancer 2009;115:2447-52.

35. Davenport K, Timoney AG, Keeley FX, et al. A 3-year review of the British Association of Urological Surgeons Section of endourology laparoscopic nephrectomy audit. BJU Int 2006;97:333-7.

36. Taub DA, Miller DC, Cowan JA, et al. Impact of surgical volume on mortality and length of stay after nephrectomy. Urology 2004;63:862-7.

37. Birkmeyer JD, Siewers AE, Finlayson EV, et al. Hospital volume and surgical mortality in the United States. N Engl J Med 2002;346:1128-37.

38. Couapel JP, Bensalah K, Bernhard JC, et al. Is there a volumeoutcome relationship for partial nephrectomy? World J Urol 2014;32:1323-9.

39. Abouassaly R, Finelli A, Tomlinson GA, et al. Volumeoutcome relationships in the treatment of renal tumors. $J$ Urol 2012;187:1984-8.

40. Toren P, Abouassaly R, Timilshina N, et al. Results of a national population-based study of outcomes of surgery for renal tumors associated with inferior vena cava Thrombus. Urology 2013;82:572-8.

41. Keoghane SR, Keeley FX, Timoney AG, et al. The British Association of Urological Surgeons Section of Endourology audit of laparoscopic nephrectomy. BJU Int 2004;94:577-81.

42. Monn MF, Bahler CD, Flack CK, et al. The impact of hospital volume on postoperative complications following robot-assisted partial nephrectomy. J Endourol 2014;28:1231-6.

43. Withington JM, Charman SC, Armitage JN, et al. Hospital volume does not influence the safety of percutaneous nephrolithotomy in England: a Population-Based Cohort Study. J Endourol 2015;29:899-906.

44. LaRiviere CA, McAteer JP, Huaco JA, et al. Outcomes in pediatric surgery by hospital volume: a population-based comparison. Pediatr Surg Int 2013;29:561-70.

45. Eskander A, Merdad M, Irish JC, et al. Volume-outcome associations in head and neck Cancer treatment: a systematic review and metaanalysis. Head Neck 2014;36:1820-34

46. Strom JB, Wimmer NJ, Wasfy JH, et al. Association between operator procedure volume and patient outcomes in percutaneous coronary intervention: a systematic review and meta-analysis. Circ Cardiovasc Qual Outcomes 2014;7:560-6. 
47. Hata T, Motoi F, Ishida M, et al. Effect of Hospital volume on Surgical Outcomes after Pancreaticoduodenectomy. Ann Surg 2016;263:664-72.

48. Boogaarts $\mathrm{HD}$, van Amerongen $\mathrm{MJ}$, de Vries $\mathrm{J}$, et al. Caseload as a factor for outcome in aneurysmal subarachnoid hemorrhage: a systematic review and meta-analysis. J Neurosurg 2014;120:605-11.

49. Luft HS, Hunt SS, Maerki SC. The volume-outcome relationship: practice-makes-perfect or selective-referral patterns? Health Serv Res 1987;22:157-82.

50. Herr HW. A history of partial nephrectomy for renal tumors. J Urol 2005;173:705-8.

51. Corman JM, Penson DF, Hur K, et al. Comparison of complications after radical and partial nephrectomy: results from the National Veterans Administration Surgical Quality Improvement Program. BJU Int 2000;86:782-9.

52. Hollenbeck BK, Taub DA, Miller DC, et al. Use of nephrectomy at select medical centers--a case of follow the crowd? J Urol 2006;175:670-4.

53. Nuttall $\mathrm{M}$, Cathcart $\mathrm{P}$, van der Meulen $\mathrm{J}$, et al. A description of radical nephrectomy practice and outcomes in England: 1995-2002. BJU Int 2005;96:58-61.
54. Fedeli U, Novara G, Alba N, et al. Trends from 1999 to 2007 in the surgical treatments of kidney Cancer in Europe: data from the Veneto Region, Italy. BJU Int 2010;105:1255-9.

55. Birkmeyer JD, Stukel TA, Siewers AE, et al. Surgeon volume and operative mortality in the United States. N Engl J Med 2003;349:2117-27.

56. Brusselaers N, Mattsson F, Lagergren J. Hospital and surgeon volume in relation to long-term survival after oesophagectomy: systematic review and meta-analysis. Gut 2014;63:1393-400.

57. Sahni NR, Dalton M, Cutler DM, et al. Surgeon specialization and operative mortality in United States: retrospective analysis. BMJ 2016;354:i3571.

58. Birkmeyer JD, Sun Y, Wong SL, et al. Hospital volume and late survival after Cancer surgery. Ann Surg 2007;245:777-83.

59. Gooiker GA, van Gijn W, Wouters MW, et al. Systematic review and meta-analysis of the volume-outcome relationship in pancreatic surgery. Br J Surg 2011;98:485-94.

60. Berger DH, Ko CY, Spain DA, et al. Society of University Surgeons position statement on the volume-outcome relationship for surgical procedures. Surgery 2003;134:34-40. 